\title{
FINITE NONNILPOTENT GROUPS WITH FEW HEIGHTS OF NONNORMAL SUBGROUPS
}

\author{
YAKOV BERKOVICH \\ University of Haifa, Israel
}

\begin{abstract}
The number of prime factors of the order of a group $G$ (multiplicities counted) is said to be the height of $G$ and denoted by $\mathrm{n}_{\lambda}(G)$. We classify the nonnilpotent groups $G$ with $\mathrm{n}_{\lambda}(G)=2$ and nonsolvable groups $G$ with $\mathrm{n}_{\lambda}(G) \in\{3,4\}$.
\end{abstract}

This note supplements [B1, 22 and $\S 3]$.

A group is said to be Dedekindian if all its subgroups are normal. It is natural to consider groups having a few nonnormal subgroups as close to Dedekindian. There is a number of papers about groups with few nonnormal subgroups (see [B1, P, S2, S3, Z]). For example, O. Schmidt [S2, S3] has studied the groups with at most two conjugate classes of nonnormal subgroups.

Let $G$ be a group (only finite groups are considered and we use the same notation as in [B1]), $n$ a natural number. Let $\lambda(n)$ be the number of prime factors of $n$ (multiplicities counted). For example, $\lambda(1)=0, \lambda(48)=5$. Set $\lambda(G)=\lambda(|G|)$, where $|G|$ is the order of $G$. The number $\lambda(G)$ we call the height of $G$. Write

$$
\mathcal{N}_{\lambda}(G)=\{\lambda(H) \mid H \text { is not normal subgroup of } \mathrm{G}\}, n_{\lambda}(G)=\left|\mathcal{N}_{\lambda}(G)\right| .
$$

We have

$$
\begin{aligned}
& \lambda\left(\mathrm{S}_{4}\right)=4, \quad \mathcal{N}_{\lambda}\left(\mathrm{S}_{4}\right)=\{1,2,3\}, \quad n_{\lambda}\left(\mathrm{S}_{4}\right)=3, \\
& n_{\lambda}(\operatorname{SL}(2,5))=4=\mathrm{n}_{\lambda}(\operatorname{PSL}(2,7))=\mathrm{n}_{\lambda}(\operatorname{PGL}(2,7))=\mathrm{n}_{\lambda}(\operatorname{PGL}(2,5)) \\
& =\mathrm{n}_{\lambda}\left(\operatorname{PSL}\left(2,3^{2}\right)\right)=\mathrm{n}_{\lambda}\left(\operatorname{PSL}\left(2,3^{3}\right)\right), \\
& \mathrm{n}_{\lambda}(\mathrm{SL}(2,7))=5 \text {. }
\end{aligned}
$$

The group $G$ is Dedekindian if and only if $\mathrm{n}_{\lambda}(G)=0$.

2000 Mathematics Subject Classification. 20D25.

Key words and phrases. Height, solvable group, Carter subgroup. 
Let $\Delta(G)$ be the set of orders of nonnormal subgroups of $G$. For example, $\Delta\left(\mathrm{S}_{4}\right)=\{2,3,4,6,8\}, \Delta(\mathrm{SL}(2,5))=\{3,5,4,6,10,8,12,20,24\}$. Obviously, $|\Delta(G)| \geq \mathrm{n}_{\lambda}(G)$. If $G$ is a $p$-group then $|\Delta(G)|=\mathrm{n}_{\lambda}(G)$ (I think that there are few nonnilpotent groups $G$ satisfying $\left.|\Delta(G)|=\mathrm{n}_{\lambda}(G)\right)$.

The groups $G$ with $\mathrm{n}_{\lambda}(G)=1$ were classified in [B1, Proposition 2.1, Theorems 2.5 and 2.6]; see also $[\mathrm{P}]$ and $[\mathrm{Z}]$ for another approach. The classification of such $p$-groups is fairly nontrivial (see above three papers). Next, in [B1, Theorem 3.1] the nonnilpotent groups $G$ with no $(G)=\mid\{|H| \mid$ $H$ is not normal in $G\} \mid=2$ are classified. In this note we classify the non primary groups $G$ with $\mathrm{n}_{\lambda}(G)=2$ (a group $G$ is said to be primary if its order is a power of a prime); see Proposition 2 and Theorem 3 . We have noticed that, as a rule, $\mathrm{n}_{\lambda}(G)<\operatorname{no}(G)$, so Theorem 3 is a proper generalization of [B1, Theorem 3.1]. We also classify the nonsolvable groups $G$ with $\mathrm{n}_{\lambda}(G) \in\{3,4\}$ (see Theorems 4, 8); in the proof of Theorem 8 we use the classification of finite simple groups.

Classification of $p$-groups $G$ such that $\mathrm{n}_{\lambda}(G)=2$, is not obtained yet. If $|G|=p^{n}, n>2$, then $\mathrm{n}_{\lambda}(G) \leq n-2$. If $G$ is a $p$-group of maximal class and order $p^{n}$, then $\mathrm{n}_{\lambda}(G)=n-2$, unless $G \cong \mathrm{Q}_{2^{n}}$ where $\mathrm{n}_{\lambda}(G)=n-3$. As Passman $[\mathrm{P}]$ proved, only few $p$-groups $G$ satisfy $\mathrm{n}_{\lambda}(G)<n-2$.

Let $G$ be extraspecial of order $p^{5}$. If $G$ has a subgroup $\cong \mathrm{E}_{p^{3}}$, then $\mathrm{n}_{\lambda}(G)=2$, and if $G$ has no subgroup $\cong \mathrm{E}_{p^{3}}$, then $\mathrm{n}_{\lambda}(G)=1$ (in the last case, by Blackburn's Theorem [Bla, Theorem 4.3], we must have $p=2$ ). Indeed, $p \in \Delta(G)$. If $H<G$ is a nonnormal subgroup of order $p^{2}$, then $G^{\prime} \not \leq H$ so $H \cong \mathrm{E}_{p^{2}}$; in that case, $H \times G^{\prime} \cong \mathrm{E}_{p^{3}}$. Since $G$ has no abelian subgroup of index $p, p^{3} \notin \Delta(G)$.

In what follows, $p, q$ are distinct primes and $r$ is a prime which may be equal or not to $p$ or $q$. We write $A \cdot B$ to denote the semidirect product of $A$ and $B$ with kernel $B$.

If $G$ is a minimal nonnilpotent group, then (O. Schmidt [S1], Y. Gol'fand [G] and L. Redei $[\mathrm{R}])$

(MNN) $G=P \cdot Q$, where $P \in \operatorname{Syl}_{p}(G)$ is cyclic, $G^{\prime}=Q \in \operatorname{Syl}_{q}(G), \mathrm{Z}(G)=$ $\mho_{1}(P) \Phi(Q)=\Phi(G), G / \Phi(Q)$ is minimal nonabelian. In particular, $G$ is minimal nonabelian if and only if $Q$ is abelian. We have $|Q|=q^{b+c}$, where $b$ is the order $q$ modulo $p$ and $c \leq b / 2$ (if $b$ is odd, then $c=0$ ). If $q>2$, then $\exp (Q)=q$.

Such $G$ we call an $\mathrm{S}(p, q)$-group or S-group. If, in addition, $Q$ is abelian, then $G$ is minimal nonabelian; in that case, such $G$ is called an $\mathrm{A}(p, q)$-group or A-group.

TheOrem 1 ([B1, Proposition 2.1]). If $G$ is a nonnilpotent group with $\mathrm{n}_{\lambda}(G)=1$, then one of the following holds:

(a) $G$ is a minimal nonabelian $\{p, q\}$-group with $\left|G^{\prime}\right|=q$.

(b) $G$ is a minimal nonabelian group of order $p q^{2}$ with $\left|G^{\prime}\right|=q^{2}$. 
In Proposition 2 we classify the groups $G$ decomposable in nontrivial direct product and such that $\mathrm{n}_{\lambda}(G)=2$,

Proposition 2. Suppose that a group $G=A \times B$, where $\mathrm{n}_{\lambda}(G)=2$, $\mathrm{n}_{\lambda}(A)>0$ and $B>\{1\}$. Then $\mathrm{n}_{\lambda}(A)=1$ and $|B|=r$. More precisely, one and only one of the following holds:

(a) $A=\mathrm{M}_{\mathrm{p}^{\mathrm{n}}}, r$ is arbitrary, $\Delta(G)=\{p, p r\}$.

(b) $A=P \cdot Q$ is minimal nonabelian of order $p^{a} q$ with $\left|A^{\prime}\right|=q$. If $a>1$, then $r \neq q$; then $\Delta(G)=\left\{p^{a}, p^{a} r\right\}$. If $a=1$, then $r$ is arbitrary; then $\Delta(G)=\{p, q, p q\}$ provided $r=q$ and $\Delta(G)=\{p, p r\}$ provided $r \neq q$.

(c) $A=P \cdot Q$ is minimal nonabelian of order $p q^{2}$ with $\left|A^{\prime}\right|=q^{2}, r$ is arbitrary, $\Delta(G)=\{p, q . p r, q r\}$.

Proof. Recall that $p, q, r$ are primes and $p \neq q$. It is easy to check that groups (a)-(c) satisfy the hypothesis. Let us prove that if $G$ satisfies the hypothesis, then it is one of groups (a)-(c).

Let $A_{0}<A$ be nonnormal and $\{1\}<L \leq B$. Then $A_{0} \times L$ is not $G$ invariant since $A \cap\left(A_{0} \times L\right)=A_{0}$. It follows that $|B|=r$ and $\mathrm{n}_{\lambda}(A)=1$. The structure of $A$ is known (see Theorem 1 and [B1, Theorem 2.4]). In particular, if $A$ is not nilpotent, it is an $\mathrm{A}(p, q)$-group. If $A$ is a $p$-group, then $A \cong \mathrm{M}_{\mathrm{p}^{\mathrm{n}}}$ and $G$ satisfy the hypothesis for arbitrary $r$.

Now let $A=P \cdot Q$ be minimal nonabelian of order $p^{a} q$. If $r=q$, then $G$ has a nonnormal subgroup of order $q$. Let us prove this. The group $G$ has exactly $q+1>2$ subgroups of order $q$. If $Q_{1}<G$ is of order $q, A^{\prime} \neq Q_{1} \neq B$, then $Q_{1}$ is not normal in $G$. Indeed, if this is false, then $\mathrm{C}_{G}\left(Q_{1}\right) \geq A B=G$, and so $B Q_{1} \leq \mathrm{Z}(G)$. In that case, $G$ is abelian, a contradiction. Besides, $G$ has a nonnormal subgroups $P$ of order $p^{a}$ and $P \times B$ of order $p^{a} q$. It follows from $\mathrm{n}_{\lambda}(G)=2$ that then $a=1$ so $G$ is as in (b).

If $A=P \cdot Q$ is minimal nonabelian of order $p q^{2}$, then $r$ is arbitrary and $\Delta(G)=\{p, q, p r, q r\}$ so $G$ satisfies the hypothesis.

In view of Proposition 2, one can confine in the sequel to nonnilpotent groups which are not decomposed in nontrivial direct product.

Note that if $G$ is an $\mathrm{S}(p, 2)$-group, then $G^{\prime} \not \mathrm{D}_{8}$. Indeed, assume that this is false; then $G^{\prime}$ has a characteristic subgroup $L$ of order 4. If $P \in \operatorname{Syl}_{p}(G)$, then $P$ centralizes $L$ and $G^{\prime} / L$ so $P$ centralizes $G^{\prime}$. It follows that $G$ is nilpotent, a contradiction.

Suppose that all proper subgroups of a nonsolvable group $G$ are solvable. Let $N<G$ be maximal normal subgroup of $G$ and $A<G$ be maximal in $G$. Then $A N$ is solvable since $A$ and $N$ are solvable. It follows that $A N<G$ so $N<A$, and we conclude that $N=\Phi(G)$. Now it is clear that $G / N$ is nonabelian simple. 
Recall that if $N$ is a normal abelian $p$-subgroup of $G, N \leq P \in \operatorname{Syl}_{p}(G)$ and $N$ is complemented in $P$, then $N$ is complemented in $G$ (Gaschütz; [H, Hauptsatz I.17.4(a)]).

In what follows, we use the above three facts freely.

Now we are ready to prove our main result.

THEOREM 3. Let $G$ be a nonnilpotent group which has no nontrivial direct factor. Let $\mathrm{n}_{\lambda}(G)=2$. Then one of the following holds:

(a) $G$ is an $\mathrm{A}(p, q)$-group of order $p q^{3}, \Delta(G)=\left\{p, q, q^{2}\right\}$.

(b) $G$ is an $\mathrm{A}(p, q)$-group of order $p^{2} q^{2}, \Delta(G)=\left\{q, p^{2}, p q\right\}$.

(c) $G$ is an $\mathrm{S}(p, q)$-group of order $p q^{3}$ with nonabelian subgroup of order $q^{3}$ and exponent $q>2, \Delta(G)=\left\{p, q, p q \cdot q^{2}\right\}$.

(d) $G=\mathrm{SL}(2,3)$ is an $\mathrm{S}(3,2)$-group of order $3 \cdot 2^{3}, \Delta(G)=\{3,4,6\}$.

(e) $G=P \cdot Q$ is an $\mathrm{S}(3,2)$-group of order $3^{2} \cdot 2^{3}, G / \mho_{1}(P) \cong \mathrm{SL}(2,3)$, $\Delta(G)=\{4,9,12,18\}$.

(f) $G=(P \times Q) \cdot R$ is a Frobenius group of order pqr ${ }^{s}$ which kernel $R$ of order $r^{s}$ is a minimal normal subgroup, $s \leq 3, p \neq r \neq q$, where $|P|=p,|Q|=q$. If $s=3$, then $P R$ and $Q R$ are minimal nonabelian and $\Delta(G)=\left\{p, q, r, r^{2}, p q\right\}$. If $s=1$, then $\Delta(G)=\{p, q, p q\}$. If $s=2$, then $\mathcal{N}_{\lambda}(G)=\{1,2\}$ (there are few possibilities for $\Delta(G)$ ).

(g) $G=P \cdot Q$ is a Frobenius group of order $p^{2} q^{s}$ with minimal normal subgroup of order $q^{s}, s=1,2,3$. If $s=3$, then the subgroup $\mho_{1}(P) Q$ of order $p q^{3}$ is minimal nonabelian. If $s=1$, then $\Delta(G)=\left\{p, p^{2}\right\}$. If $s=2$, then $\mathcal{N}_{\lambda}(G)=\{1,2\}$. If $s=3$, then $\Delta(G)=\left\{p, p^{2}, q, q^{2}\right\}$.

(h) $G=P \cdot Q$, where $P \cong \mathrm{Q}_{8},|Q|=q, G$ contains exactly one cyclic subgroup of index $2, \Delta(G)=\{4,8\}$.

(i) $G=P \cdot(Q \times R)$ of order $p^{a} q r, P \in \operatorname{Syl}_{p}(G)$ is cyclic of order $p^{a}$, $|Q|=q,|R|=r, p \neq r \neq q, \mho_{1}(P)=\mathrm{Z}(G), G / \mathrm{Z}(G)$ is a Frobenius group of order pqr, $\Delta(G)=\left\{p^{a}, p^{a} q, p^{a} r\right\}$.

(j) $G=P \cdot Q$ is a Frobenius group of order $p q^{2}$ with minimal normal subgroup of order $q, \mathcal{N}_{\lambda}(G)=\{1,2\}$.

(k) $G=P \cdot Q$ is of order $p^{a} q, P$ is cyclic of order $p^{a}, a>1, G / \mathrm{Z}(G)$ is a Frobenius group of order $p^{2} q, \Delta(G)=\left\{p^{a-1}, p^{a}\right\}$.

(l) $G=P \cdot Q$ is of order $p^{a} q^{2}, a>1, \mho_{1}(P)=\mathrm{Z}(G), G / \mho_{1}(P)$ is a Frobenius group of order $p q^{2}$, all subgroups of order $q$ are $G$-invariant, $\Delta(G)=\left\{p^{a}, p^{a} q\right\}$.

Proof. Write $\mathcal{N}_{\lambda}(G)=\{m . n\}, m<n$.

(i) Let $G=P \cdot Q$ be an $\mathrm{S}(p, q)$-group as in $(\mathrm{MNN})$.

(i1) Suppose that $P$ is not maximal in $G$. Then $Q$ is nonabelian so special. If $\{1\}<Z \leq \mathrm{Z}(Q)$, then $P Z$ is not normal in $G$, so $|\mathrm{Z}(Q)|=q$, and we have $|P|=p^{m}$ and $n=m+1$. Next, $|Q / \mathrm{Z}(Q)|=q^{b}$, where $b>1$ is the order of $q$ $(\bmod p)$. Therefore, if $\mathrm{Z}(Q)<Q_{1}<Q$, then $Q_{1}$ and $\mho_{1}(P) Q_{1}$ are not normal in $G$. Since $\mathrm{n}_{\lambda}(G)=2$, then $b \leq 3$. Since $G$ has a nonnormal subgroup of 
order $q^{2}$, we get $m \leq 2$. Since $G$ has a nonnormal subgroup of order $q^{b}$, we get $b \leq n$.

If $m=1$, then $n=2$ so $b=2$ and $|G|=p q^{3}$, where $Q$ is nonabelian of order $q^{3}, Q \in\left\{\mathrm{Q}_{8}, \mathrm{~S}\left(p^{3}\right)\right\}$, where $\mathrm{S}\left(p^{3}\right)$ is a group of order $p^{3}$ and exponent $p>2$. If $Q \cong \mathrm{Q}_{8}$, then $p=3, \Delta(G)=\{3,4,6\}$ and $G \cong \mathrm{SL}(2,3)$ is as in (d). If $q>2$, then $\Delta(G)=\left\{p, q, p q, q^{2}\right\}$ and $G$ is as in (c).

Now let $m=2$. If $b=3$, then $Q$ has a non- $G$-invariant subgroups of orders $q, q^{2}$ and $q^{3}$ so $\mathrm{n}_{\lambda}(G)>2$, a contradiction. Thus, $b=2$ and so $|G|=p^{2} q^{3}$. If $q>2$, then $Q$ has, in addition, a nonnormal subgroup of order $q$ so $\mathcal{N}_{\lambda}(G)=\{1,2,3\}$, a contradiction. Thus, $q=2, Q \cong Q_{8}, p=3$ so $|G|=3^{2} 2^{3}, \Delta(G)=\{4,9,12,18\}$ and $G$ is as in (e).

(i2) Suppose that $P$ is maximal in $G$. Then $Q$ is minimal normal in $G$ and $G$ is minimal nonabelian. Set $|Q|=q^{b}$; then $b>1$ (otherwise, $\mathrm{n}_{\lambda}(G)=1$ ). Since $G$ has a nonnormal subgroup of order $q$, we get $m=1$. Since $\mathrm{n}_{\lambda}(G)=2$, we get $b \leq 3$. Therefore, if $|P|=p$, then $b=3,|G|=p q^{3}, \Delta(G)=\left\{p, q, q^{2}\right\}$, and $G$ is as in (a).

Now let $|P|>p$; then $|P|=p^{n}$. If $Q_{1}<Q$ is of order $q$, then $\Omega_{1}(P) Q_{1}$ is not normal in $G$ so $n=\lambda\left(\Omega_{1}(P) Q_{1}\right)=2$ and $\mathcal{N}_{\lambda}(G)=\{1,2\}$. Then $b \leq 3$. If $b=3$ and $Q_{2}<Q$ is of order $q^{2}$, then $\Omega_{1}(P) Q_{2}$ is not normal in $G$ so $3 \in \mathcal{N}_{\lambda}(G)$, a contradiction. Thus, $b=2,|G|=p^{2} q^{2}, \Delta(G)=\left\{q, p^{2}, p q\right\}$ so $G$ is as in (b).

Next we assume that $G$ is not an S-group.

(ii) Let us prove that $G$ is solvable. Assume that $G$ is a counterexample of minimal order. If $H<G$, then $\mathrm{n}_{\lambda}(H) \leq \mathrm{n}_{\lambda}(G) \leq 2$ so all proper subgroups of $G$ are solvable, by induction. If $N \triangleleft G$, then $\mathrm{n}_{\lambda}(G / N) \leq \mathrm{n}_{\lambda}(G) \leq 2$ so all proper epimorphic images of $G$ are solvable. It follows that $G$ is nonabelian simple and $\lambda(H) \leq 2$ for all $H<G$. Let $p$ be a minimal prime divisor of $G$ and $P \in \operatorname{Syl}_{p}(G)$; then $|P|>p$ (Burnside). If $|P|>p^{2}$, then $G$ contains subgroups of orders $p, p^{2}$ and $p^{3}$ so $\mathrm{n}_{\lambda}(G)>2$, a contradiction. Thus, $|P|=p^{2}$ and $\mathcal{N}_{\lambda}(G)=\{1,2\}$. By Burnside, $P<\mathrm{N}_{G}(P)$ and so $\lambda\left(\mathrm{N}_{G}(P)\right)>2$, a contradiction.

Since $G$ is solvable, there is in $G$ a Carter subgroup $K$. Recall that $K$ is nilpotent and $\mathrm{N}_{G}(K)=K$ (so $\mathrm{Z}(G)<K$ ), and all Carter subgroups are conjugate in $G$, and, whenever $K \leq H \leq G$, then $\mathrm{N}_{G}(H)=H$ [H, Satz VI.12.3]. By hypothesis, $K<G$. We conclude that $K$ is either maximal or second maximal in $G$.

(iii) Assume that $K$ is not primary. Then $K=P \times Q$, where $P \in \operatorname{Syl}_{p}(K)$ is not $G$-invariant and $Q>\{1\}$. Since $\mathrm{n}_{\lambda}(G)=2, K$ is maximal in $G$ and $m=\lambda(P), n=\lambda(K)$. Since all proper subgroups of $P$ are $G$-invariant, it follows that $P$ is cyclic. If $|Q|$ is not prime and $\{1\}<Q_{0}<Q$, then $P Q_{0}$ is not $G$-invariant so $\mathrm{n}_{\lambda}(G)>2$, a contradiction. Thus, $|Q|=q$ so $K$ is cyclic, $|K|=p^{m} q, n=m+1$ and $\mathcal{N}_{\lambda}(G)=\{m, m+1\}$. 
(iii1) Let $m>1$; then $Q \triangleleft G$ since $\lambda(Q)=1<m$. In that case, consideration of $G$-invariant subgroup $\mathrm{C}_{G}(Q) \geq K$ shows that $Q \leq \mathrm{Z}(G)$. Similarly, $\mho_{1}(P) \leq \mathrm{Z}(G)$ so $|K: \mathrm{Z}(G)|=p$. We have $K \cap K^{x}=\mathrm{Z}(G)$ for all $x \in G-K$, and so $\bar{G}=G / \mathrm{Z}(G)=\bar{K} \cdot \bar{N}$ is a Frobenius group with kernel $\bar{N}$ of order $r^{b}$ $(r \neq p)$ and index $p$. Since $K$ is maximal in $G, \bar{N}$ is minimal normal subgroup of $\bar{G}$ so $\bar{G}$ is $\mathrm{A}(p, r)$-group. If $b>1$, then $\bar{N}$ has a non- $G$-invariant subgroup $\bar{F}$ of order $r^{b-1}$; then $\lambda(F)=\lambda(\mathrm{Z}(G))+b-1=m+b-1 \leq n=m+1$, and we conclude that $b=2$. If $r \neq q$ and $b=2$, then $G$ has a nonnormal subgroup of order $r$, and we get $\mathrm{n}_{\lambda}(G)>2$ since $\lambda(r)=1<m$, a contradiction. Thus, if $b=2$, then $r=q$.

Suppose that $r=q$. Let $Q_{0} \in \operatorname{Syl}_{q}(G)$; then $\left|Q_{0}\right|=q^{b+1}$ and $Q_{0} / Q \cong \mathrm{E}_{q^{b}}$; next, $Q_{0} \triangleleft G$ since $N \triangleleft G$ and $Q_{0}$ is a direct factor of $N$. The subgroup $Q_{0}$ is noncyclic (otherwise, by (MNN), $G$ has no S-subgroup so nilpotent). Since $m>1$ and $\mathcal{N}_{\lambda}(G)=\{m, m+1\}$, all subgroups of order $q$ are normal in $G$. Assume that $\exp \left(Q_{0}\right)=q$. If $\left|Q_{0}\right|=q^{2}$, then $Q$ is a direct factor of $G$ (Maschke's Theorem), contrary to the hypothesis. Thus, $\left|Q_{0}\right|>q^{2}$ so $G$ has a nonnormal subgroup of order $q^{2}$, and we conclude that $m=2$; then $\mathcal{N}_{\lambda}(G)=\{2,3\}$. By the previous paragraph, $b=2$ so $\left|Q_{0}\right|=q^{3}$. All subgroups of order $q$ are $G$-invariant so $\exp \left(Q_{0}\right)=q^{2}$. If $q>2$, then $\Omega_{1}\left(Q_{0}\right)\left(\cong \mathrm{E}_{q^{2}}\right)$ is normal in $G$ so $\bar{N}$ is not minimal normal subgroup of $\bar{G}$, a contradiction. Thus, $q=2$ so that $Q_{0} \cong \mathrm{Q}_{8}$; then $p=3$. In that case, $|P|=3^{2},|G|=3^{2} 2^{3}$, $G / \mho_{1}(P) \cong \mathrm{SL}(2,3)$ and $\Delta(G)=\{4,9,12,18\}$ so $G$ is as in (e).

Now let $r \neq q$. Then, by the first paragraph of this part, $b=1$ and $G=K \cdot R=(P \times Q) \cdot R=(P \cdot R) \times Q$, contrary to the hypothesis.

(iii2) Now let $m=1$ so $|K|=p q$; then $\mathcal{N}_{\lambda}(G)=\{1,2\}$. Since $K$ is maximal in $G$, we get $\mathrm{N}_{G}(P)=K$ so $P \in \operatorname{Syl}_{p}(G)$.

Assume that $Q$ is not normal in $G$; then $\mathrm{N}_{G}(Q)=K$ so $K$ is a $\{p, q\}$ Hall subgroup of $G$. By Burnside's Normal $p$-Complement Theorem applied twice, $G=K \cdot H$, where $H$ is a minimal normal subgroup of $G$ which is a $\{p, q\}^{\prime}$-Hall subgroup of $G$. It follows that $|H|=r^{s}$, where $r \in \pi(G)-\{p, q\}$. We have $s \leq n+1=3$. We see that $G$ is a Frobenius group with kernel $H$. Assume that $s=3$. Then the subgroups $P H$ and $Q H$ are minimal nonabelian. Indeed, assume that, for example, $P H$ is not minimal nonabelian. Then, by Maschke's Theorem, it contains a non- $G$-invariant subgroup of order $p r^{2}$ so $3 \in \mathcal{N}_{\lambda}(G)$, a contradiction. In that case, $\Delta(G)=\left\{p, q, r, p q, r^{2}\right\}$ so $\mathrm{n}_{\lambda}(G)=2$. If $s=1$, then $\Delta(G)=\{p, q, p q\}$. If $s=2$, then this and previous two groups are as in (f) (for $\Delta(G)$ we have few possibilities, among of them $\{p, q, p q, r\},\{p, q, p q, p r, r\},\{p, q, p q, q r, r\},\{p, q, p q, p r, q r, r\})$.

Now suppose that $Q \triangleleft G$. Then, as above, $Q=\mathrm{Z}(G), G / Q=\bar{G}=\bar{K} \cdot \bar{N}$ is a Frobenius group with kernel $\bar{N}$, where $|\bar{K}|=p$ and $|\bar{N}|=r^{b}, r \neq p, \bar{N}$ is a minimal normal subgroup of $\bar{G}$. Since $G$ has no nontrivial direct factor, we get $r=q$ so $G=P \cdot N$ and $N \in \operatorname{Syl}_{q}(G)$. In that case, $N$ is noncyclic (otherwise, by (MNN), $G$ has no S-subgroup so nilpotent). Since $Q$ is not a direct factor 
of $G$, we get $b>1$ (Fitting's Lemma). Since $\bar{G}$ has a nonnormal subgroup $\bar{Q}_{1}$ of order $q^{b-1}$, then $b=\lambda\left(Q_{1}\right)=2$ (recall that 2 is the maximal member of the set $\left.\mathcal{N}_{\lambda}(G)\right)$; then $|N|=q^{3}$. Since $N / Q$ is minimal normal subgroup of $G / Q$, we get $\left|\Omega_{1}(N)\right| \neq q^{2}$. If $\left|\Omega_{1}(N)\right|=q$, then $q=2, N \cong \mathrm{Q}_{8}$ and $G \cong \operatorname{SL}(2,3)$ is as in (d). If $\left|\Omega_{1}(N)\right|=q^{3}$, then $N$ is nonabelian (otherwise, $Q$ is a direct factor of $G$, by Fitting's Lemma) so $N \cong \mathrm{S}\left(q^{3}\right)$, and $G$ is as in (c).

(iv) Let $K$ be a $p$-subgroup with non- $G$-invariant maximal subgroup, say $K_{1}$; then $K \in \operatorname{Syl}_{p}(G)$ is maximal in $G, \mathcal{N}_{\lambda}(G)=\{m, m+1\},|K|=p^{m+1}$, $m \geq 1$. In that case, if $L<K$ is of index $>p$, then $L \triangleleft G$ and so $K$ is not generated by subgroups of index $p^{2}$. Then one of the following holds:

1) $K$ is cyclic;

2) $K$ is abelian of type $\left(p^{m}, p\right)$;

3) $K \cong \mathrm{Q}_{8}$.

Note that the group $\mathrm{M}_{p^{m+1}}$ is also not generated by subgroups of index $p^{2}$, however, if $K \cong \mathrm{M}_{p^{m+1}}, m>1$, then $K$ has a nonnormal subgroup of order $p$ which is impossible since $\lambda(p)=1<m$.

(iv1) Let $K$ be abelian of order $p^{m+1}$; then $G$ has a normal $p$-complement $H$ (Burnside), and $H$ is a minimal normal subgroup of $G$ since $K$ is maximal in $G$. Set $|H|=q^{b}$. If $K$ is abelian of type $(p, p)$, then $G$ is not a Frobenius group so $\mathrm{C}_{G}\left(P_{1}\right)=G$ for some $P_{1}<P$ of order $p$. In that case, $P_{1}$ is a direct factor of $G$, a contradiction.

Suppose that $b=1$. If $K$ is cyclic, then $\Delta(G)=\left\{p^{m}, p^{m+1}\right\}, G / \mathrm{Z}(G)$ is a Frobenius group of order $p^{2} q$. Now let $K$ be abelian of type $\left(p^{m}, p\right)$. In that case, as we have proved, $m>1$. Then all subgroups of order $p$ are $G$-invariant so lie in $\mathrm{Z}(G)$. Therefore, if $K=U \times V$, where $|V|=p$, then $V$ is a direct factor of $G$ (Gaschütz), a contradiction. Thus, $K$ is cyclic so $G$ is as in (k).

Next we assume that $b>1$. Since $G$ has nonnormal subgroups of order $q^{i}, i=1, \ldots, b-1$, we get $b \leq 3$ and $m=1,|K|=p^{2}$. By the above, $K$ is cyclic. In that case, $G$ is a Frobenius group of order $p^{2} q^{b}, 1<b \leq 3$. If $b=2$, then $\Delta(G)=\left\{p, q, p^{2}\right\}$ and $G$ is as in $(\mathrm{g})$. Let $b=3$. Then $\mho_{1}(K) H$ must be minimal nonabelian (otherwise, $G$ contains a subgroup of order $p q^{2}$, by Maschke's Theorem), which is $q$-closed so non- $G$-invariant, and we get $p, p^{2}, p q^{2} \in \mathcal{N}_{\lambda}(G)$ so $\mathrm{n}_{\lambda}(G)>2$, a contradiction. Thus, $G$ is as in (g).

(iv2) Let $K \cong \mathrm{Q}_{8}$; then $\mathcal{N}_{\lambda}(G)=\{2,3\}$. We claim that then $G=K \cdot Q$ is 2-nilpotent, where $|Q|=q^{b}$. Indeed, at least two maximal subgroups of $K$ are nonnormal in $G$, the subgroup $L<K$ of order 2 is $G$-invariant. By Burnside, $Q L / L \triangleleft G / L$. Since $|Q L|=2|Q|, Q$ is characteristic in $Q L$ so $Q \triangleleft G$. Since $K$ is maximal in $G, Q$ is minimal normal subgroup of $G$. If $|Q|>q$, there is a nonnormal subgroup of order $q$ in $Q$ so $1 \in \mathcal{N}_{\lambda}(G)$, a contradiction. Thus, $b=1$. In that case, $\mathrm{C}_{G}(Q)$ is cyclic of index 2 in $G$ so $G$ is as in (h).

(v) Suppose that all maximal subgroups of $K$ are normal in $G$. Then $K$ is a cyclic $p$-subgroup, and we conclude that $K \in \operatorname{Syl}_{p}(G)$ since $\mathrm{N}_{G}(K)=K$, 
and hence $G=K \cdot H$, where $H$ is a normal $p^{\prime}$-Hall subgroup of $G$ (Burnside). Then $|H|$ is not a prime (otherwise, $\mathrm{n}_{\lambda}(G)=1$ ). Since $\mathrm{C}_{G}\left(\mho_{1}(K)\right) \geq K H=$ $G$, we get $\mho_{1}(K)=\mathrm{Z}(G)$. Write $\bar{G}=G / \mho_{1}(G)$; then $\bar{G}=\bar{K} \cdot \bar{H}$ is a Frobenius group so $\bar{H}$ is nilpotent, by theorem of Witt [BZ, Theorem 10.7]. Since $\bar{H} \cong H$, the subgroup $H$ is also nilpotent.

(v1) Suppose that $|\pi(H)|>1$; then $\pi(H)=\{q, r\}$ (otherwise, $K<L<$ $M<G$ and $L, M$ are not normal in $G$ so $\mathrm{n}_{\lambda}(G)>2$ (here we use Hall's theorem on solvable groups). In that case, $\{p, q\}$ - and $\{p, r\}$-Hall subgroups of $G$, say $U$ and $V$, respectively, are not normal in $G$. It follows that $\lambda(U)=$ $\lambda(V)$. Obviously, $U$ and $V$ are maximal in $G$ (otherwise, $\mathrm{n}_{\lambda}(G)>2$ ). Let $Q \in \operatorname{Syl}_{q}(U)$ and $R \in \operatorname{Syl}_{r}(V)$; then $H=Q \times R$ and $Q, R$ are minimal normal subgroups of $G$ (for example, $K Q$ is maximal in $(K Q) \cdot R=G$ so $R$ is a minimal normal subgroup of $G)$. We have $|K|=p^{m}$ and $\lambda(K Q)=n$.

Let $|Q|>q$. Then $G$ has a nonnormal subgroup of order $q$ so $\lambda(K)=$ $m=1$ (otherwise, $1, \lambda(K), \lambda(K Q) \in \mathcal{N}_{\lambda}(G)$ so $\mathrm{n}_{\lambda}(G)>2$ ). In that case, $G$ has a nonnormal subgroup of order $q r$ so $p, q r,|K Q| \in \Delta(G)$ and $\mathrm{n}_{\lambda}(G)>2$, a contradiction. Thus, $|Q|=q$ so $|H|=q r,|G|=p^{m} q r, \bar{G}=G / \mho_{1}(K)$ is a Frobenius group of order $p q r$ with kernel $\bar{H}$ of order $q r$, and we get $\Delta(G)=\left\{p^{m}, p^{m} q, p^{m} r\right\}$, and $G$ is as in (i).

In what follows we assume that $H \in \operatorname{Syl}_{q}(G)$.

(v2) Suppose that $K<M<G$, where $M$ is maximal in $G$; then $K$ is maximal in $M$ (otherwise, $\mathrm{n}_{\lambda}(G)>2$ ). We have $M=K \cdot Q$ (Burnside) and $\mho_{1}(K)=\mathrm{Z}(G)$. Set $|Q|=q^{b},|G: M|=q^{s}=|H: Q|,|K|=p^{m}$. Next, $Q$ is a minimal normal subgroup of $M$ and so of $G$ (in fact, $\mathrm{N}_{G}(Q)>M$ whence $Q \triangleleft G)$.

(v2.1) Suppose that $m>1$. Then $b=1$ (otherwise, there is in $G$ the following non- $G$-invariant subgroups: $K$ of order $p^{m}>p, K Q$ of order $p^{m} q^{b}>$ $p^{m}$ and a subgroup of $Q$ of order $q$ so $\mathrm{n}_{\lambda}(G)>2$ ).

Let, in addition, $s>1$; then $m=2$. Indeed, since $H / Q$ contains a non- $G$-invariant subgroup $Q_{1} / Q$ of order $q$, we get $\lambda\left(Q_{1}\right)=2<\lambda(K Q)$ so $2=\lambda\left(Q_{1}\right)=\lambda(K)=m$. Since $p^{2}, p^{2} q \in \Delta(G)$ and $\mathrm{n}_{\lambda}(G)=2$, we must have $\mathcal{N}_{\lambda}(G)=\{2,3\}$. It follows that $s \leq 3$ (otherwise, $H / Q$ contains a non- $G$ invariant subgroup $Q_{2} / Q$ of order $q^{3}$ so $\left.\lambda\left(Q_{2}\right)=4>3\right)$ and all subgroups of order $q$ must be $G$-invariant since $1 \notin \mathcal{N}_{\lambda}(G)$.

Assume that $s=3$. Then $|H|=q^{4}$ and $H$ has a non- $G$-invariant subgroup $H_{1}$ of order $q^{3}$. Then $\mho_{1}(K) \times H_{1}$ of order $p q^{3}$ is not $G$-invariant, a contradiction since $\lambda\left(\mho_{1}(K) H_{1}\right)=4>3$. Thus, $s<3$.

Assume that $s=2$. Then $|H|=q^{3}$. It follows that $\exp (H)=q^{2}$ (otherwise, $H=\Omega_{1}(H)$ is elementary abelian and all subgroups of order $q$ in $H$ are $G$-invariant, which is not the case since $H / Q \cong \mathrm{E}_{q^{2}}$ is minimal normal subgroup of $G / Q$ ). Then $K$ is contained in (non- $G$-invariant) subgroup of order $p^{2} q^{2}$, a contradiction since $4 \notin \mathcal{N}_{\lambda}(G)$. 
It remains to consider case $s=1$ (by assumption, $m>1$ ); then $|H|=q^{2}$, $|G|=p^{m} q^{2}, \mathcal{N}_{\lambda}(G)=\{m, m+1\}$. All subgroups of $G$ of order $q$ are $G$ invariant, $G / \mho_{1}(K)$ is a Frobenius group of order $p q^{2}, \Delta(G)=\left\{p^{m}, p^{m} q\right\}$. Since $H$ is cyclic or abelian of type $(p, p)$, we get two nonisomorphic groups. Our $G$ are as in (1).

(v2.2) Let $m=1$. Then $G$ is a Frobenius group and $b \leq 2$ (indeed, if $b>2$, then $G$ has a nonnormal subgroups of orders $p, q^{2}$ and $p q^{b}$ so $\mathrm{n}_{\lambda}(G)>2$, a contradiction).

Suppose that $b=1$. Then $p, p q \in \Delta(G)$ so $\mathcal{N}_{\lambda}(G)=\{1,2\}$ hence $s \leq 2$ (if $s>2$, then $G$ has a non- $G$-invariant subgroup of order $q^{3}$ so $\mathrm{n}_{\lambda}(G)>2$ ). If $s=1$, then $G$ (of order $p q^{2}$ ) is as in (j). Let $s=2$. Then $G$ has no subgroup of order $p q^{2}$ (otherwise, $\mathrm{n}_{\lambda}(G)>2$ ). It follows that $H$ is either $\cong \mathrm{Q}_{8}$ (then $G \cong \operatorname{SL}(2,3))$ or $\exp (H)=p$. In the first case, $K$ is not a Carter subgroup since it centralized by $Q$, a contradiction. In the second case, $q \equiv 1(\bmod p)$ since $K Q$ is nonnilpotent so $H / Q$ is not a minimal normal subgroup of $G / Q$ (take in $G / Q$ a minimal nonnilpotent subgroup!), a contradiction.

Suppose that $b=2$. Then $K$ and $K Q$ are not $G$-invariant so $\mathcal{N}_{\lambda}(G)=$ $\{1,3\}$ and $s \leq 2$ (otherwise, $G$ has a nonnormal subgroup of order $q^{4}$ so $4 \in$ $\mathcal{N}_{\lambda}(G)$, a contradiction. If $s=1$, then the $G$-invariant subgroup $[H, Q]<Q$ so $Q \leq \mathrm{Z}(H)$ since $Q$ is a minimal normal subgroup of $G$, and we conclude that $H$ is abelian. If $\exp (H)=q^{2}$, then $\{1\}<\Phi(H)<Q$, a contradiction since $Q$ is a minimal normal subgroup of $G$. Thus, $H \cong \mathrm{E}_{q^{3}}$. Since $G / Q$ is nonabelian of order $p q$, we get $q \equiv 1(\bmod p)$. Then $Q$ is not minimal normal subgroup of $K Q$ (indeed, by (MNN), $K Q$ contains a proper S-subgroup) so $Q$ is not minimal normal subgroup of $G$ since $H$ is abelian, a final contradiction.

It follows from the proof of Theorem 3 that if $\mathrm{n}_{\lambda}(G)=2$, then $|\Delta(G)| \leq 6$, and this estimate is attained. Therefore, Theorem 3 is a very strong generalization of [B1, Theorem 3.1].

It is easy to deduce from Theorems 1 and 3 classification of nonnilpotent groups without three nonnormal subgroups of pairwise distinct orders and which are not nontrivial direct products. We get the following groups of Theorems 1 and 3: 1(a), 1(b), 3(g) of order $p^{2} q, 3(\mathrm{~h}), 3(\mathrm{j}), 3(\mathrm{k}), 3(\mathrm{l})$ (compare with [B1, Theorem 3.1]). Note that O. Schmidt has classified the groups with one [S1] and two [S2] non-invariant classes of conjugate subgroups (the proof of the last result is not full; see $[\mathrm{B} 1, \S 2, \S 3])$.

In what follows we use freely the following facts. If $\lambda(G) \leq 3$, then $G$ is solvable. If $G$ is nonsolvable, there is $H<G$ with $\lambda(H) \geq 3$. Let us prove the second assertion using induction on $|G|$. Then all proper subgroups of $G$ are solvable so $G / \Phi(G)$ is nonabelian simple. By induction, $\Phi(G)=\{1\}$. Let $p$ be the minimal prime divisor of $|G|$ and $P \in \operatorname{Syl}_{p}(G)$; then $P$ is noncyclic (Burnside). By assumption, $\lambda(P)=2$ so $P$ is abelian. Again, by Burnside, 
$\mathrm{N}_{G}(P)>P$. If $H / P \leq \mathrm{N}_{G}(P)$ is of prime order, then $\lambda(H)=3$ so $G$ is not a counterexample.

We also use freely the description of subgroups of the simple group $\operatorname{PSL}\left(2, p^{n}\right)[\mathrm{D}]$.

THEOREM 4. If $G$ is a nonsolvable group and $\mathrm{n}_{\lambda}(G)=3$, then one of the following holds:

(a) $G \cong P S L(2,5)$.

(b) $G \cong P S L(2, p), \lambda(p \pm 1) \leq 3, p^{2} \not \equiv 1(\bmod 5),|G| \not \equiv 0(\bmod 8)$.

Proof. (i) Suppose that $G$ is a nonabelian simple. Note that a nonsolvable group contains a subgroup $S$ with $\lambda(S)=3$. It follows that $G$ has no proper subgroup $H$ with $\lambda(H)>3$. Thus, all proper subgroups $H$ of $G$ are solvable and $\lambda(H) \leq 3$. In what follows we do not use the Odd Order Theorem. Let $p$ be the minimal prime divisor of $|G|$ and $P \in \operatorname{Syl}_{p}(G)$; then $P$ is noncyclic (Burnside). By what has just has been said, $p^{2} \leq|P| \leq p^{3}$.

(i1) Let $|P|=p^{3}$. Let $H<G$ be an $\mathrm{S}(q, p)$-subgroup $(H$ exists, by Frobenius Normal $p$-Complement Theorem). Then $\lambda(H)=3$ so $p=2$ and $H \cong \mathrm{A}_{4}$, the alternating group of degree 4. Let $P_{1} \in \operatorname{Syl}_{p}(H)$. One may assume that that $P_{1}<P$. Then $\mathrm{N}_{G}\left(P_{1}\right) \geq\langle P, H\rangle$ so $\lambda\left(\mathrm{N}_{G}\left(P_{1}\right)\right)>3$, contrary to the previous paragraph.

(i2) Let $|P|=p^{2}$; then $p=2$. In that case, $G \cong \operatorname{PSL}(2, p)$, by [W]. Since all subgroups of $G$ are known [D], it follows that $G$ is as in (b).

Next we assume that $G$ is not simple. Let $M<G$ be a maximal normal subgroup. Then $G / M$ is simple.

(ii) Suppose that $M$ is solvable; then $\bar{G}=G / M$ is nonabelian simple so, by Theorem $3, \mathrm{n}_{\lambda}(\bar{G})=3$. Let $\bar{H}<\bar{G}$ and $\bar{F}<\bar{G}$, where $\lambda(\bar{H})=1$ and $\lambda(\bar{F})=3$. Since all nonidentity subgroups of $\bar{F}$ are not $\bar{G}$-invariant, it follows that $\mathcal{N}_{\lambda}(G)=\{\lambda(M)+1, \lambda(M)+2, \lambda(M)+3\}$. Then all proper subgroups of $H$ are normal in $G$ so $H$ is a cyclic $q$-subgroup for prime $q=|\bar{H}|$. In particular, $M$ is a cyclic $q$-subgroup. As $q$ one can choose every prime from $\pi(\bar{G})$. Since $|\pi(\bar{G})|>2$ (Burnside), we get a contradiction.

(iii) Suppose that $M$ is nonsolvable. Then $\mathrm{n}_{\lambda}(M)>2$ (Theorem 3) so $\mathrm{n}_{\lambda}(M)=3$ since $\mathrm{n}_{\lambda}(M) \leq \mathrm{n}_{\lambda}(G)=3$. It follows that all subgroups of the simple group $G / M$ are normal so $|G: M|=q$, a prime. By induction, $M$ is a group from conclusion. It follows that $\mathcal{N}_{\lambda}(M)=\{1,2,3\}=\mathcal{N}_{\lambda}(G)$. Let $P \in \operatorname{Syl}_{2}(M)$. Then, by Frattini's Lemma, $G=M \mathrm{~N}_{G}(P)$. Since $\mathrm{N}_{M}(P)>P$, we get $\lambda\left(\mathrm{N}_{G}(P)\right)>3$, and this is a final contradiction since all subgroups of $\mathrm{N}_{G}(P)$, containing $P$, are not normal in $G$.

I do not know if the number of groups of Theorem 4(b) is infinite.

Let $\mathrm{n}_{s \lambda}(G)=\mid\{\lambda(H) \mid H<G$ is nonnormal and solvable $\} \mid$. Arguing as in the proof of Theorem 4 , one can prove that if $\mathrm{n}_{s \lambda}(G)=3$, then a nonsolvable 
group $G$ is as in Theorem 4 but in (b) the condition $p^{2} \not \equiv 1(\bmod 5)$ must be omitted.

REMARK 5. Here we consider a nonnilpotent group $G$ all of whose nonnormal subgroups are cyclic. ${ }^{1}$ Suppose that $G$ has a noncyclic Sylow subgroup. By hypothesis, all noncyclic Sylow subgroups are $G$-invariant. If $P \in \operatorname{Syl}_{p}(G)$ is noncyclic, then $P \triangleleft G$ and $G / P$ is Dedekindian. It follows that $G$ is solvable (this is also true if $P$ does not exist, by Burnside). Since $G$ is nonnilpotent, $P$ is the unique noncyclic Sylow subgroup of $G$. Since all Sylow subgroups of the Dedekindian group $G / P$ are cyclic, $G / P$ is itself cyclic, and we get $G^{\prime} \leq P$. Let $K$ be a Carter subgroup of $G$. Then $K$ is cyclic and maximal in $G$ since, if $K<M<G$, then $M$ is noncyclic so normal in $G$, which is impossible. It follows that $G=K P$ so $P_{0}=K \cap P \triangleleft G$. Since $K \leq \mathrm{C}_{G}\left(P_{0}\right) \triangleleft G$, we conclude that $P_{0} \leq \mathrm{Z}(G)<K$. Write $\bar{G}=G / \mathrm{Z}(G)$. Then $\bar{G}=\bar{K} \cdot \bar{P}$ is a Frobenius group with cyclic complement $\bar{K}$ and kernel $\bar{P}$ which is a minimal normal subgroup of $\bar{G}$. Since $\bar{P}$ is elementary abelian, it has no proper subgroup of order $p^{2}$ so $|\bar{P}| \leq p^{2}$. Suppose that $|\bar{P}|=p^{2}$ and $P_{0}>\{1\}$. If $P_{1} / P_{0}<P / P_{0}$ is of order $p$, then $P_{1}$ is cyclic since it is not normal in $G$. It follows that $P$ has $p+1$ distinct cyclic subgroups of index $p$; note that $\mathrm{Z}(G)$ is cyclic. Since $P$ is noncyclic, we get $P \cong \mathrm{Q}_{8}$ hence $\left|P_{0}\right|=2,|\bar{K}|=3$. If $Z_{0}$ is a subgroup. of index 2 in $\mathrm{Z}(G)$, then $G / Z_{0} \cong \operatorname{SL}(2,3)$. As it is easy to see, such $G$ satisfies the hypothesis. Now let $P_{0}=\{1\}$. Then $P \cong \mathrm{E}_{p^{2}}, G=K \cdot P$. We have $K_{G}=\mathrm{Z}(G)$. Write $\bar{G}=G / \mathrm{Z}(G)$. Since $K$ is cyclic and maximal in $G$, the group $G$ satisfies the hypothesis if and only if for every $\bar{H} / \bar{P}$ of prime order, $\bar{H}$ is minimal nonabelian. If $|\bar{P}|=p$, then $G$ satisfies the hypothesis.

REMARK 6. Suppose that all subgroups of prime order $p>2$ are $G$ invariant. Then $G^{\prime}$ is $p$-nilpotent. Indeed, all normal cyclic subgroups of $G$ centralize $G^{\prime}$. It follows from $(\mathrm{MNN})$ that $G^{\prime}$ has no $\mathrm{S}(q, p)$-subgroups so $p$-nilpotent, by Frobenius' Normal $p$-Complement Theorem. In particular, if all subgroups of odd prime orders are $G$-invariant, then $G^{\prime} / P$ is nilpotent for $P \in \operatorname{Syl}_{2}(G)$.

REMARK 7 . In the proof of Theorem 8 we use the following fact: If all subgroups of $G$ of order 4 are normal, then $G$ is solvable. Isaacs in his letter at $4 / 08 / 07$ noticed that, under this condition, either $G$ is 2-nilpotent or its Sylow 2 -subgroup is normal and elementary abelian and has proved this. Below we offer another proof of Isaacs' assertion. Assume that $G$ is not 2-nilpotent. Let $P_{1} \in \mathrm{Syl}_{2}(G)$. By Frobenius' Normal $p$-Complement Theorem, there is in $G$ an $\mathrm{S}(q, 2)$-subgroup $S=Q \cdot P$; then $\exp (P) \leq 4$, by (MNN). If $L<P$ is cyclic of order 4 , then $L$ centralizes $G^{\prime}>P$, a contradiction since $\exp (\mathrm{Z}(P))=2$. Thus, $\exp (P)=2$; then $P \cap \mathrm{Z}(S)=\{1\}$. If $|P|>4$, it has two subgroups

\footnotetext{
${ }^{1}$ The $p$-groups all of whose nonnormal subgroups are cyclic, are almost classified in $[\mathrm{P}$, Theorem 2.9].
} 
$A, B$ of order 4 such that $A \cap B$ is of order 2. Then $\{1\}<A \cap B \leq P \cap \mathrm{Z}(S)$, a contradiction. Thus, $|P|=4$ so $P$ is minimal normal subgroup in $S$. Assume that $P<U \leq P_{1}$, where $|U: P|=2$. Then $U$ contains a subgroup $X \neq P$ of order 4. In that case, $P \cap X$ is of order 2 and contained in $\mathrm{Z}(S) \cap P$, a contradiction. Thus, if $G$ is not 2-nilpotent, its Sylow 2-subgroup is normal in $G$ and $\cong \mathrm{E}_{4}$.

THEOREM 8. Let $G$ be a nonsolvable group with $\mathrm{n}_{\lambda}(G) \leq 4$. Then one of the following holds:

(a) $G \cong \operatorname{PSL}(2, p)$, where $\lambda(p \pm 1) \leq 4$.

(b) $G \cong \operatorname{PSL}(2,8)$.

(c) $G \cong \operatorname{PSL}\left(2,3^{2}\right) \cong \mathrm{A}_{6}$.

(d) $G \cong P S L\left(2,3^{3}\right)$.

(e) $G=G_{1} \times C$, where $G_{1}$ is a group of Theorem 4 and $|C|$ prime.

(f) $G \cong \operatorname{PGL}(2, p)$, where either $p=5,7$ or $p$ is as in Theorem $4(b)$.

(g) $G \cong \mathrm{SL}(2, p)$, where $p$ is such as in Theorem 4 .

Proof. (i) (This part is proved by Kazarin) Suppose that $G$ is nonabelian simple; then $\lambda(H) \leq 4$ for all solvable $H<G$. Let $\{1\}<R<G$ be primary. Set $N=\mathrm{N}_{G}(R)$. Assume that $N$ is nonsolvable. Then there is a solvable $F / R<N / R$ with $\lambda(F / R)>2$ (Theorem 4). Since all subgroups of (the solvable subgroup) $F$ are nonnormal in $G$, it follows that $\lambda(R)=1$ and $\lambda(F)=$ 4. Then $N / R \cong P S L(2, p)$ is as in Theorem 4 so $\{1,2,3,4, \lambda(N)\} \subseteq \mathcal{N}_{\lambda}(G)$, a contradiction since $\lambda(N)>4$. Thus, all local subgroups of $G$ are solvable. By Thompson's Theorem [T], $G$ is isomorphic with one of the following groups:

(1) $P S L\left(2, p^{n}\right), S z\left(2^{2 m+1}\right), P S L(3,3), P S U(3,3), A_{7}, M_{11},{ }^{2} F_{4}(2)^{\prime}$.

Since Sylow 2-subgroups of $G$ have order at most 16, this excludes groups $\mathrm{Sz}\left(2^{2 m+1}\right)$ and ${ }^{2} F_{4}(2)^{\prime}$. Note, that if the order of a Sylow 2-subgroup $S$ of $G$ is 16 , then $S$ is maximal in $G$. Hence $\mathrm{M}_{11}, \operatorname{PSL}(3,3), \operatorname{PSU}(3,3)$ and $\mathrm{A}_{7}$ does not satisfy the hypothesis. Now it remains to discuss groups $\operatorname{PSL}\left(2, p^{n}\right)$ only. Note that $\operatorname{PSL}\left(2, p^{n}\right)$ contains the subgroup $\cong \operatorname{PSL}(2, p)$.

(i1) Let $G \cong \operatorname{PSL}\left(2,2^{n}\right), n>1$. Then $G$ has a solvable subgroup $H$ of order $2^{n}\left(2^{n}-1\right)$. Since $4 \geq \lambda(H)=n+\lambda\left(2^{n}-1\right)$, we get $n \leq 3$. If $n=2$, then $G \cong \operatorname{PSL}(2,4) \cong \mathrm{A}_{5}$; then $\mathrm{n}_{\lambda}(G)=3$. If $n=3$, then $G \cong \operatorname{PSL}(2,8)$ and $\mathrm{n}_{\lambda}(G)=4$.

(i2) Let $G \cong \operatorname{PSL}\left(2, p^{n}\right), n>1, p>2$. Then $G$ has a solvable subgroup $H$ of order $\frac{1}{2}\left(p^{n}-1\right) p^{n}$. We have $n-1+\lambda\left(p^{n}-1\right)=\lambda(H) \leq 4$.

Let $p=3$. Since $\lambda\left(3^{n}-1\right) \geq 2$, we get $n \leq 3$. If $n=2$, then $G \cong$ $\operatorname{PSL}\left(2,3^{2}\right) \cong \mathrm{A}_{6}$. If $n=3$, then $G \cong \operatorname{PSL}\left(2,3^{3}\right)$. Both these groups satisfy the hypothesis.

Now let $p>3, n>1$. Since $\lambda\left(\frac{1}{2}\left(p^{n}-1\right)\right) \geq 2$, we must have $n=2$. Then $\lambda\left(\frac{1}{2}\left(p^{2}-1\right)\right) \geq 3$ so $\lambda\left(\frac{1}{2} p^{n}\left(p^{n}-1\right)\right)=n+\lambda\left(\frac{1}{2}\left(p^{2}-1\right)\right)>4$, contrary to the hypothesis. 
(i3) Let $G \cong \operatorname{PSL}(2, p)$. If $F<G$ is nonsolvable, then $F \cong P S L(2,5)$ so $\lambda(F)=4$. If $H<G$ is solvable, then $\lambda(H) \leq 4$. Since all subgroups of $G$ are known, we get $\lambda(p \pm 1) \leq 4$. The case where $G$ is simple, is complete.

(ii) Suppose that the Fitting subgroup $\mathrm{F}(G)>\{1\}$. Let $R$ be the solvable radical of $G$; then $G / R$ has no nonidentity solvable normal subgroup. Write $a=\lambda(R)$. If $H / R<G / R$ is solvable, then $\lambda(H / R) \leq 4$ (otherwise, $\left.\mathrm{n}_{\lambda}(G / R)>4\right)$ and all nonidentity subgroups of $H / R$ are not $G$-invariant.

Let $H / R<G / R$ with $\lambda(H / R)=3$ (such $H$ exists, by Theorem 4). Then $a+1, a+2, a+3 \in \mathcal{N}_{\lambda}(G)$. By Remarks 6 and $7, G$ has a nonnormal subgroups of orders $r>2$ and 4 . It follows that $a=1$ and so $\mathcal{N}_{\lambda}(G)=\{1,2,3,4\}$ so $|R|=p$, a prime. In that case, $\mathrm{n}_{\lambda}(G / R)=3$ so $G / R$ is a group of Theorem 4 ; in particular, $G$ is nonabelian simple. Then $\mathrm{C}_{G}(R)=G$ so $R=\mathrm{Z}(G)$. If $R<G^{\prime}$, then $R$ is a subgroup of the Schur multiplier of the group $G / R$ so $G \cong \operatorname{SL}(2, p)$, where either $p=5$ or $p$ is as in Theorem 4(b) (Schur [Sc]). If $R \not \leq G^{\prime}$, then $G=G_{1} \times R$, where $G_{1}$ is as in Theorem 4 .

(iii) Now suppose that $\mathrm{F}(G)=\{1\}$ and $G$ is not simple; then $G$ has no nonidentity solvable normal subgroup. By assumption, $\lambda(H) \leq 4$ for all solvable $H<G$. Let $N$ be a maximal normal subgroup of $G$. Then, by assumption, $N$ is nonsolvable.

Assume that $G / N$ is nonabelian simple. Let $P \in \operatorname{Syl}_{2}(N)$ and $K=$ $\mathrm{N}_{G}(P)$. We have $|P| \geq 4$ and all nonidentity subgroups of $P$ and $K / P$ are not $G$-invariant. It follows that $K / P$ has no solvable subgroup $F / K$ such that $\lambda(F / K)>2$. Then, by Theorem $4, K / P$ is solvable so $\lambda(K) \leq 4$. However, $N K=G$ (Frattini's Lemma) so $K$ cover the nonsolvable group $G / N$, and we conclude that $K$ is nonsolvable, a contradiction.

Thus, $|G / N|=p$, a prime, so $G^{\prime} \leq N$. Assume that $G^{\prime}<N$; then $\lambda\left(G / G^{\prime}\right) \geq 2$. Let $P \in \operatorname{Syl}_{2}\left(G^{\prime}\right)$; then $\lambda\left(\overline{\mathrm{N}}_{G^{\prime}}(P)\right) \geq 3$ (Burnside) and $\mathrm{N}_{G^{\prime}}(P)$ is solvable (here we use the Odd Order Theorem) so $\lambda\left(\mathrm{N}_{G}(P)\right) \geq 5$. Since $\mathrm{N}_{G}(P)$ is solvable (Frattini's Lemma), we get $\mathrm{F}(G)>\{1\}$, a contradiction. Thus, $\left|G: G^{\prime}\right|=p$ so that $G^{\prime}=N$. The same argument shows that $G^{\prime \prime}=G^{\prime}$, i.e., $G^{\prime}$ is the last member of the derived series of $G$. Let $R$ be a minimal normal subgroup of $G$. Then $R \leq G^{\prime}$ and, as above, $G / R$ is solvable so $R=G^{\prime}$. It follows that $R=R_{1} \times \cdots \times R_{k}$, where $R_{1} \cong \ldots \cong R_{k}$ are nonabelian simple. In that case, $R$ has a solvable subgroup $H$ such that $4 \geq \lambda(H)=3 k$ so $k=1$ since $\mathrm{F}(G)=\{1\}$. Thus, $R$ is nonabelian simple. If $\lambda(H) \leq 3$ for all solvable $H<R$, then $R$ is a group of Theorem 4 , and then $G \cong \operatorname{PGL}(2, p)$, where either $p=5$ or $p$ is as in Theorem 4(b) (recall that in the case under consideration, $\operatorname{Aut}(R) \cong \operatorname{PGL}(2, p))$.

In what follows we assume that there is a solvable $H<R$ with $\lambda(H)=4$. Since the normalizer of every nonidentity solvable subgroup of $R$ is solvable, $R$ is one of groups of list (1). As in (i), we have only to check the case where $R \cong \operatorname{PSL}\left(2, p^{n}\right)$. If $p=2$, then $R \cong \operatorname{PSL}(2,8)$. In that case, if $P \in \operatorname{Syl}_{2}(R)$, then $\mathrm{N}_{R}(P)=C \cdot P$ is of order $2^{3} \cdot 7$ so $\mathrm{N}_{G}(P)=2^{3} \cdot 7 p$ (in fact, $p=3$ since 
$\mid \operatorname{Aut}\left(\operatorname{PSL}((2,8): \operatorname{PSL}(2,8) \mid=3)\right.$. Since $\mathrm{N}_{G}(P)$ is solvable and $\lambda\left(\mathrm{N}_{G}(P)\right)=5$, we get $\mathrm{F}(G)>\{1\}$, a contradiction. If $p=3$, then $R \cong \operatorname{PSL}\left(2,3^{n}\right), n \in\{2,3\}$. Let $Q \in \operatorname{Syl}_{3}(\mathrm{R})$. Assume that $n=2$. Then $\lambda\left(\mathrm{N}_{\mathrm{R}}(\mathrm{Q})\right)=4$ so $\lambda\left(\mathrm{N}_{\mathrm{G}}(\mathrm{Q})\right)=5$ and, as above, we get a contradiction since $\mathrm{N}_{G}(Q)$ is solvable. Now let $n=3$. Then $\lambda\left(\mathrm{N}_{\mathrm{R}}(\mathrm{Q})\right)=4$ so $\lambda\left(\mathrm{N}_{\mathrm{G}}(\mathrm{Q})\right)=5$, and we again get a contradiction.

Thus, $p>3$. In that case, $n=1$ and $\operatorname{Aut}(R) \cong \operatorname{PGL}(2, p)$ so $G \cong$ $\operatorname{PGL}(2, p)$. Assume that $H<R$ is dihedral of order $p \pm 1$ with $\lambda(p \pm 1)=4$. Then $\lambda\left(\mathrm{N}_{G}(H)\right)=5, \mathrm{~N}_{G}(H)$ is solvable so $\mathrm{F}(G)>\{1\}$, a contradiction. Thus, $p$ is such as in Theorem 4, completing the proof.

I think that if $|\Delta(G)|=\mathrm{n}_{\lambda}(G)$, then $|\pi(G)|$ must be small.

Let no $(G)$ be as above. Then, if $G$ is nonsolvable, then $\operatorname{no}(G) \geq \lambda(G)+$ $|\pi(G)| \geq 7[\mathrm{~B} 2]$.

\section{Problems}

1. Classify the $p$-groups $G$ satisfying $\mathrm{n}_{\lambda}(G)=2$.

2. Classify the nonnilpotent groups $G$ satisfying $\mathrm{n}_{\lambda}(G)=3$.

3. Classify the nonsolvable groups $G$ satisfying $\mathrm{n}_{\lambda}(G)=5$.

4. Let $\mathrm{n}_{s \lambda}(G)$ is defined in the paragraph preceding Remark 5. Classify the nonsolvable groups $G$ satisfying $\mathrm{n}_{s \lambda}(G) \in\{4,5\}$.

5. Classify the groups $G$ such that $\mathrm{n}_{s \lambda}(G) \leq|\pi(G)|$.

6. Classify the groups all of whose minimal nonabelian subgroups (Ssubgroups) have the same order.

7. Classify the groups with $|\Delta(G)|=\mathrm{n}_{\lambda}(G)$.

8. Classify the $p$-groups all of whose nonnormal subgroups are metacyclic (abelian).

9. Find $\mathrm{n}_{\lambda}\left(\mathrm{A}_{n}\right), \mathrm{n}_{\lambda}\left(\mathrm{S}_{n}\right)$.

ACKNOWLEDGEMENTS.

I am indebted to Lev Kazarin and Martin Isaacs for classification of simple groups in Theorem 8 and more exact statement of the main result of Remark 7 , respectively.

\section{REFERENCES}

[B1] Y. Berkovich, Nonnormal and minimal nonabelian subgroups of a finite group, submitted.

[B2] Y. Berkovich, Some criteria for the solvability of finite groups, Sibirsk. Mat. Z̆. 4 (1963), 723-728.

[BZ] Ya. G. Berkovich and E. M. Zhmud, Characters of Finite Groups. Part 1, Translations of Mathematical Monographs 172, American Mathematical Society, Providence, RI, 1998.

[Bla] N. Blackburn, Generalizations of certain elementary theorems on p-groups, Proc. London Math. Soc. (3) 11 (1961), 1-22.

[D] L. E. Dickson, Linear groups with an exposition of the Galois field theory, Dover Publications, Inc., New York, 1958.

[H] B. Huppert, Endliche Gruppen. I, Springer-Verlag, Berlin-New York, 1967. 
[G] Yu. A. Gol'fand, On groups all of whose subgroups are special, Doklady Akad. Nauk SSSR 60 (1948), 1313-1315.

[P] D. S. Passman, Nonnormal subgroups of p-groups, J. Algebra 15 (1970), 352-370.

[R] L. Redei, Die endlichen einstufig nichtnilpotenten Gruppen, Publ. Math. Debrecen 4 (1956), 303-324.

[S1] O. Schmidt, Groups all of whose subgroups are nilpotent, Mat. Sb. 31 (1924), 366-372.

[S2] O. Schmidt, Groups having only one class of nonnormal subgroups, Mat. Sb. 33 (1926), 161-172.

[S3] O. Schmidt, Groups with two classes of nonnormal subgroups, Proc. Seminar on group theory (1938), 7-26.

[Sc] I. Schur, Über die Darstellung der endlichen Gruppen durch gebrochene lineare Substitutionen, J. Reine Angew. Math. 127 (1904), 20-50.

[T] J. G. Thompson, Nonsolvable finite groups all of whose local subgroups are solvable, Bull. Amer. Math. Soc. 74 (1968), 383-437.

[W] J. H. Walter, The characterization of finite groups with abelian Sylow 2-subgroup, Ann. Math. (2) 89 (1969), 405-514.

[Z] G. Zappa, Finite groups in which all nonnormal subgroups have the same order, Atti Accad. Naz. Lincei Cl. Sci. Fis. Mat. Natur. Rend. Lincei (9) Mat. Appl. 13 (2002), 5-16; II, ibid 14 (2003), 13-21.

Y. Berkovich

Department of Mathematics

University of Haifa

Mount Carmel, Haifa 31905

Israel

Received: 13.8.2007.

Revised: 15.6.2008. 